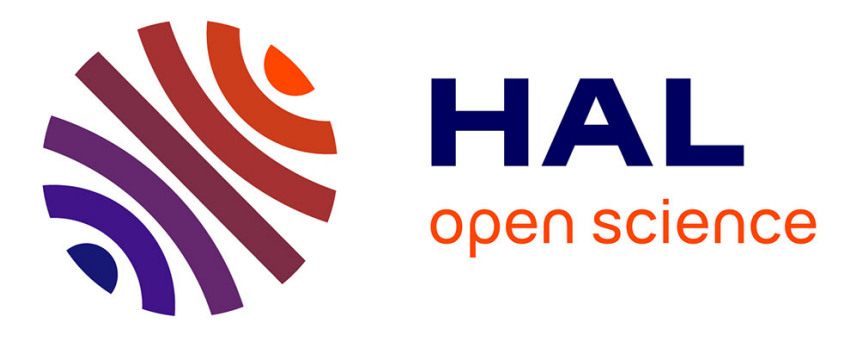

\title{
Les céréales à paille hôtes de Meloidogyne naasi Franklin. III. - Recherches de sources de résistance parmi les espèces voisines du blé tendre
} Françoise Person-Dedryver, Joseph Jahier, Danielle Pannetier

\section{To cite this version:}

Françoise Person-Dedryver, Joseph Jahier, Danielle Pannetier. Les céréales à paille hôtes de Meloidogyne naasi Franklin. III. - Recherches de sources de résistance parmi les espèces voisines du blé tendre. Agronomie, 1985, 5 (7), pp.573-578. hal-00884786

\section{HAL Id: hal-00884786 https://hal.science/hal-00884786}

Submitted on 1 Jan 1985

HAL is a multi-disciplinary open access archive for the deposit and dissemination of scientific research documents, whether they are published or not. The documents may come from teaching and research institutions in France or abroad, or from public or private research centers.
L'archive ouverte pluridisciplinaire HAL, est destinée au dépôt et à la diffusion de documents scientifiques de niveau recherche, publiés ou non, émanant des établissements d'enseignement et de recherche français ou étrangers, des laboratoires publics ou privés. 


\title{
Les céréales à paille hôtes de Meloidogyne naasi Franklin. III. - Recherches de sources de résis- tance parmi les espèces voisines du blé tendre
}

\author{
Françoise PERSON-DEDRYVER \& Joseph JAHIER $\left({ }^{*}\right)$ \\ avec la collaboration technique de Danielle PANNETIER \\ I.N.R.A., Laboratoire de Zoologie \\ (*) Station d'Amélioration des Plantes, Centre de recherches de Rennes, F-35650 Le Rheu
}

RÉSUMÉ

\begin{abstract}
Le comportement vis-à-vis de Meloidogyne naasi de 3 espèces de blés diploïdes et de 7 espèces d'Aegilops a été étudié sur sol naturellement infesté. A l'intérieur de chaque espèce, on observe une variation dans le caractère hôte. Les 12 lignées de blés diploïdes testées sont toutes multiplicatrices de $M$. naasi ; cependant chez $T$. monococcum ssp. monococcum, le développement de femelles est toujours moindre. Les Aegilops : kotschyi, speltoides, triaristata, uniaristata, ventricosa sont de bons hôtes du parasite. Par contre, les Aegilops umbellulata et variabilis présentent des lignées très résistantes. L'intérêt et le transfert dans le blé des gènes de résistance de ces dernières sont discutés.
\end{abstract}

Mots clés additionnels : Nématode, Triticum spp., Aegilops spp., gènes de résistance, introgression.

Cereals as hosts of Meloidogyne naasi Franklin. III. - Investigations into the level of resistance of wheat relatives.

The behaviour towards Meloidogyne naasi of 3 diploid wheat species and 7 Aegilops species was studied on infested soil. Within each species, variation in the " host character » is observed. The 12 lines of diploid wheat tested were all susceptible to $M$. naasi but development of females was less on those belonging to T. monococcum ssp. monococcum. Certain Aegilops species (kotschyi, speltoides, triaristata, uniaristata, ventricosa) were good hosts of the parasite while lines of Ae. umbellulata and Ae. variabilis were very resistant. The value of transferring their resistance genes into wheat is discussed.

Additional key words : Nematode, Triticum spp., Aegilops spp., resistance genes, introgression.

\section{INTRODUCTION}

En France, les céréales à paille et les graminées fourragères subissent des dommages importants consécutifs aux attaques de Meloidogyne naasi Frankl. qui induit des galles sur les racines (CAUBEL et al., 1972 ; BAYON 1978 ; RIVOAL et al., 1983). L'emploi de variétés d'orge ou de blé résistantes s'opposant au développement des femelles du nématode paraît être la méthode de lutte la plus attrayante, car elle est facile d'utilisation et économiquement rentable en permettant l'intensification des cultures céréalières. Or actuellement, aucune des variétés de blé ou d'orge cultivées en France ne présente une résistance vis-à-vis du parasite (PERSON-DEDRYVER, 1985).
La recherche de sources de résistance a été entreprise en Grande Bretagne par COOK \& YORK (1981) et concerne plus de 10000 lignées d'Hordeum vulgare L. appartenant aux collections britanniques, américaines et turques. 4 lignées, dont une provenant de Turquie, présentent un niveau de résistance intermédiaire ; la lignée turque a une résistance qui dépend d'un système génétique récessif difficile à utiliser dans des programmes de sélection (COOK et al., 1981). La recherche de résistance s'est orientée chez les espèces voisines du blé et de l'orge cultivée dans le but d'obtenir de bons géniteurs de résistance à $M$. naasi. COOK \& YORK (1981) détectent 2 lignées d'Hordeum chilense L. et une d'Hordeum jubatum L. ne permettant pas la multiplication du nématode et signalent qu'en 
sa présence, Aegilops variabilis Eig. est indemne de galles (COOK et al., 1976). Des travaux américains (RoBERTs et al., 1982), concernant 5 espèces d'Aegilops, 2 de blé diploïdes et 3 de blés tétraploïdes, montrent qu'il n'est pas possible de trouver parmi elles des résistances vis-à-vis de $M$. naasi. Cependant, une lignée d'Ae. squarrosa L. d'origine afghane est résistante à 4 pathotypes de $M$. incognita (Kofoid \& White) Chitwood et à 4 populations de $M$. javanica (Treub) Chitwood, 2 espèces de nématodes qui ne sont pas signalées comme nuisibles aux cultures céréalières en France.

La culture du blé étant prédominante dans les assolements céréaliers français, nous nous sommes surtout attachés à rechercher des sources de résistance aux nématodes au sein des espèces voisines. L'amélioration de la résistance chez le blé tendre est envisagée à partir de l'exploitation d'hybrides interspécifiques et intergénériques. Au Rheu, la recherche de gènes de résistance à Heterodera avenae Woll., nématode à kyste des céréales, a déjà été entreprise par DOSBA \& RIVOAL (1981) qui ont mis en évidence la résistance d'Ae. ventricosa Tausch., Ae. uniaristata Vis., Ae. variabilis et le bon comportement de la lignée d'addition disomique blé $-A e$. ventricosa lignée $n^{\circ} 11$.

Des lignées de 2 espèces de blés diploïdes et de 7 espèces d'Aegilops comprenant les 3 résistantes à H. avenae (DOSBA \& RIVOAL, 1981) ont été semées sur sol naturellement infesté en $M$. naasi. Les formations de femelles, œufs et galles du nématode, estimées suivant une méthode mise au point préalablement (PERSON-DEDRYVER, 1984), nous renseignent sur la nature du caractère bon hôte ou résistant de chacune des lignées de ces espèces.

\section{MATÉRIEL ET MÉTHODES}

\section{A. Matériel végétal}

33 lignées appartenant à 2 espèces de blés diploïdes et à 7 espèces d'Aegilops diploüdes ou tétraploïdes sont testées vis-à-vis de $M$. naasi. Ces lignées font partie de la collection du laboratoire «Blé et Cytogénétique " de la Station d'Amélioration des Plantes. Il s'agit de :

Triticum monococcum (L.) Mk. ssp. beoticum (Boiss.) Mk. (2 n = 14, génome A) n os 1038, 1039.

ssp. monococcum L. ( $\mathrm{n}=14$, génome $\mathrm{A}) \mathrm{n}^{\mathrm{os}} 38$, $55,57,99,157,219,7553$.

Triticum urartu Tum. ( $2 \mathrm{n}=14$, génome $\left.\mathrm{A}^{\mathrm{u}}\right)$ $\mathrm{n}^{\text {os }} 7796,7797,7798$.

Aegilops speltoides Tausch $(2 \mathrm{n}=14$, génome $\mathrm{S})$ $\mathrm{n}^{\mathrm{os}} 1,3,18,25,34$.

Aegilops umbellulata Zhuk. ( $2 \mathrm{n}=14$, génome U) $\mathrm{n}^{\mathrm{os}} 1,2,3,4,5$.

Aegilops uniaristata Vis. $\left(2 \mathrm{n}=14\right.$, génome $\left.\mathrm{U}_{\mathrm{n}}\right)$ $\mathrm{n}^{\mathrm{os}} 1,2,3,4$.

Aegilops variabilis Eig. ( $2 \mathrm{n}=28$, génomes US') $\mathrm{n}^{\text {os }} 1,5,6,7$.

Aegilops kotschyi Boiss. ( $2 \mathrm{n}=28$, génome US $\left.{ }^{1}\right)$ $\mathrm{n}^{\mathrm{os}} 1,2,3,4,6$.
Aegilops ventricosa Tausch $(2 \mathrm{n}=28$, génomes $\left.\mathrm{DU}_{\mathrm{n}}\right) \mathrm{n}^{\text {os }} 7,8,10,14,21,24$.

Aegilops triaristata Willd ( $2 \mathrm{n}=28$, génomes UM) $\mathrm{n}^{\text {os }} 1,2,3,4$.

\section{B. Matériel et méthodes nématologiques}

Différents tests sont disponibles actuellement (PERSON-DEDRYVER, 1984) pour juger de la qualité d'hôte des céréales à l'égard de $M$. naasi. Mais seuls ceux réalisés et décrits sur sol naturellement infesté par le nématode permettent une détection fiable de la résistance d'espèces sauvages tells que les Aegilops et seront donc les seuls utilisés dans ce travail.

Ils consistent à stocker, dès septembre, dans des bouteilles en matière plastique de 1,5 1 renversées dont le fond a été préalablement coupé, du sol provenant de parcelles de la localité du Rheu (Ille \& Vilaine) ayant été cultivées en blé la même année. Le niveau d'infestation en $M$. naasi, estimé avant l'implantation de l'essai, est compris entre 6 et 22 larves infectieuses au $2^{\mathrm{e}}$ stade $\left(\mathrm{L}_{2}\right)$ par $\mathrm{g}$ de sol et reste, dans tous les cas, supérieur au seuil de nuisibilité connu sur blé et proche de $5 \mathrm{~L}_{2}$.

Les caryopses prégermés sont implantés dans le sol à raison d'un par bouteille, avec au minimum 4 répétitions par lignée sauf en 1980 où l'essai n'en présentait que 3. Les implantations sont toutes faites le même jour pour une même année de culture, sur une période pouvant aller de janvier à avril selon les années.

Les tests réalisés en 1979 et 1980 sont analysés début septembre alors que ceux de 1981 et 1982 le sont en juillet. L'analyse de chaque plante consiste en une notation des indices de galles définis précédemment (PERSON-DEDRYVER, 1984) et en un relevé du poids des racines.

Après broyage du système racinaire, les femelles et les œufs du nématode sont extraits par une méthode flottaison-centrifugation (COOLEN \& D'HERDE, 1972). Leurs nombres sont déterminés après observation au microscope binoculaire et rapportés au $g$ de racine pour faciliter les comparaisons entre plantes de poids racinaires très différents.

\section{Méthodes statistiques}

Le test non paramétrique de KRUSKAL \& WALLIS (HOLLANDER \& WOLFE, 1973) est utilisé pour mettre en évidence les différences statistiques existant dans la formation des femelles, œufs et galles du nématode sur différentes lignées appartenant à 1 ou plusieurs espèces cultivées la même année. Ce test est poursuivi par celui de DUNN (1964) sur les rangs afin de préciser, si nécessaire, les différences enregistrées.

\section{RÉSULTATS}

\section{A. La qualité d'hôte des blés diploüdes}

Toutes les lignées de $T$. urartu et de $T$. monococcum étudiées en 1980 et 1982 multiplient le nématode (tabl. 1). Mais en 1982, Les lignées 1038 et 1039 de 
TABLEAU 1

Formation des femelles, des œufs et des galles de Meloidogyne naasi sur des lignées de blés diploides.

Production of females, eggs and galls of Meloidogyne naasi on accessions of diploid wheats.

\begin{tabular}{|c|c|c|c|c|c|c|c|}
\hline \multirow{2}{*}{$\begin{array}{l}\text { Espèces } \\
\text { et lignées }\end{array}$} & \multirow{2}{*}{$\begin{array}{c}\text { Potentiel } \\
\text { infectieux } \\
\text { L2/g de sol }\end{array}$} & \multicolumn{2}{|c|}{ Femelles/g de racine } & \multicolumn{2}{|c|}{ OEufs/femelle } & \multirow{2}{*}{$\begin{array}{l}\text { OEufs/g } \\
\text { de racine } \\
\mathrm{m}\end{array}$} & \multirow{2}{*}{$\begin{array}{l}\text { Indice pondéré } \\
\text { de galles }\end{array}$} \\
\hline & & (p) & $r$ & $\mathrm{~m}$ & (p) & & \\
\hline
\end{tabular}

\begin{tabular}{|c|c|c|c|c|c|c|c|c|c|}
\hline ESSAI 198 & & & & & & & & & \\
\hline Triticum & & & & & & & & & \\
\hline urartu & 7796 & & 120,6 & $(23-278)$ & 3 & 63,1 & $(20-103)$ & 4436 & 56,7 \\
\hline & 7797 & & 45,3 & $(15-105)$ & 3 & 72,8 & $(26-158)$ & 5820 & 66,7 \\
\hline & 7798 & & 25,1 & $(9-40)$ & 3 & 23,7 & $(16-31)$ & 550 & 46,7 \\
\hline Triticum & & & & & & & & & \\
\hline m. beoticum & 828 & 12 & 12,8 & $(9-17)$ & 3 & 33,9 & $(1-53)$ & 480 & 33,3 \\
\hline Triticum & & & & & & & & & \\
\hline m. monococcum & $n \quad 38$ & & 12,3 & $(6-21)$ & 3 & 49,3 & $(21-82)$ & 466 & 56,7 \\
\hline & 57 & & 37,1 & $(20-47)$ & 3 & 62,4 & $(9-123)$ & 1813 & 63,3 \\
\hline & 157 & & 8,3 & $(2-16)$ & 3 & 56,6 & $(35-99)$ & 221 & 53,3 \\
\hline & 219 & & 33,2 & $(20-41)$ & 3 & 14,1 & $(10-18)$ & 466 & 70 \\
\hline $\mathrm{H}$ & & & & 13,25 & & & 8,96 & 13,28 & 8,26 \\
\hline ESSAI 198 & & & & & & & & & \\
\hline Triticum & & & & & & & & & \\
\hline m. beoticum & 1039 & & $325,1^{\mathrm{b}}$ & $(87-824)$ & 5 & $47,2^{\mathrm{ab}}$ & $(24-76)$ & 15604 & $68^{b}$ \\
\hline & 1038 & 11 & $138,2^{\mathrm{ab}}$ & $(24-234)$ & 4 & $10^{\mathrm{a}}$ & $(1-14)$ & 1975 & $50^{\mathrm{a}}$ \\
\hline Triticum & & & & & & & & & \\
\hline m. monococcum & & & $30,1^{\mathrm{a}}$ & $(11-57)$ & 6 & $92,3^{\mathrm{b}}$ & $(41-149)$ & 2885 & $53,3^{\mathrm{a}}$ \\
\hline & 99 & & $27,6^{\mathrm{a}}$ & $(15-60)$ & 6 & $107,9 \mathrm{~b}$ & $(53-154)$ & 2930 & $53,3^{a}$ \\
\hline & 7553 & & $30,1^{\mathrm{a}}$ & $(10-53)$ & 5 & $53,4^{\text {ab }}$ & $(20-121)$ & 1761 & $48^{a}$ \\
\hline $\mathrm{H}$ & & & & $14,02^{* *}$ & & & $13,74^{* *}$ & 7,93 & $24,6^{* * *}$ \\
\hline
\end{tabular}

T. monococcum ssp. beoticum donnent significativement plus de femelles par $\mathrm{g}$ de racine que les lignées 55, 99 et 7553 de $T$. monococcum ssp. monococcum. A l'inverse, les femelles formées en 1982 sur $T$. $m$. monococcum pondent en moyenne plus d'œufs que celles trouvées sur $T$. $m$. beoticum. Globalement, nous n'enregistrons donc pas de différence statistique dans le nombre d'œufs obtenus par $g$ de racine et par lignée appartenant aux 2 sous-espèces. Seule la lignée 1039 de $T$. $m$. beoticum fournit beaucoup plus de femelles et d'œufs par $\mathrm{g}$ de racine que les autres lignées et possède d'ailleurs un indice de galles significativement supérieur aux autres.

\section{B. La qualité d'hôte des Aegilops triaristata, variabi- lis, uniaristata et ventricosa}

Toutes les lignées d'Ae. uniaristata et d'Ae. ventricosa étudiées en 1982 sont de très bons hôtes du nématode (tabl. 2). Lés nombres de femelles et œufs formés par $\mathrm{g}$ de racine atteignent des maxima importants avec la lignée 7 d'Ae. ventricosa : 1583 femelles et 32089 œufs.

En 1979, les 4 lignées d'Ae. variabilis forment moins de femelles et d'œufs par g de racine et de galles par plante que les lignées d'Ae. triaristata (tabl. 2).
Les lignées 1 et 6 d'Ae. variabilis présentent même un bon niveau de résistance avec respectivement 2 et 3 plantes sur lesquelles on ne trouve aucune femelle.

\section{Etude de la résistance d'Ae. variabilis et de ses progéniteurs}

L'essai de 1981 (tabl. 3) confirme, avec un taux d'infestation supérieur en larves infectieuses $L_{2}$, le bon niveau de résistance à l'encontre de $M$. naasi trouvé en 1979 dans les lignées 1 et 6 d'Ae. variabilis (tabl. 2).

Ae. kotschyi dont les génomes sont très proches de ceux d'Ae. variabilis (FURUTA, 1981) ne fournit aucune lignée susceptible.d.avoir une résistance satisfaisante et comparable à celles des lignées 1 et 6 d'Ae. variabilis (tabl. 3).

Les 5 lignées d'Ae. speltoides, progéniteur du génome $\mathrm{S}$ d'Ae. variabilis, sont toutes d'excellents hôtes du nématode. Ae. umbellulata, à l'origine du génome U d'Ae. variabilis, présente à l'image de ce dernier des lignées «bons ou mauvais hôtes» du parasite telles que, respectivement, les lignées $n^{\circ} 4$ et $\mathrm{n}^{\circ} 3$. Les lignées 1 et 3 d'Ae. umbellulata ont un très bon niveau de résistance équivalent à celui des lignées 1 et 6 d'Ae. variabilis (tabl. 3). 
TABLEAU 2

Formation des femelles, des aeufs et des galles de Meloidogyne naasi sur des lignées d'Aegilops triaristata, Ae. uniaristata, Ae. variabilis, Ae. ventricosa.

Production of fernales, eggs and galls of Meloidogyne naasi on accessions of Aegilops triaristata, Ae. uniaristata, Ae. variabilis, Ae. ventricosa.

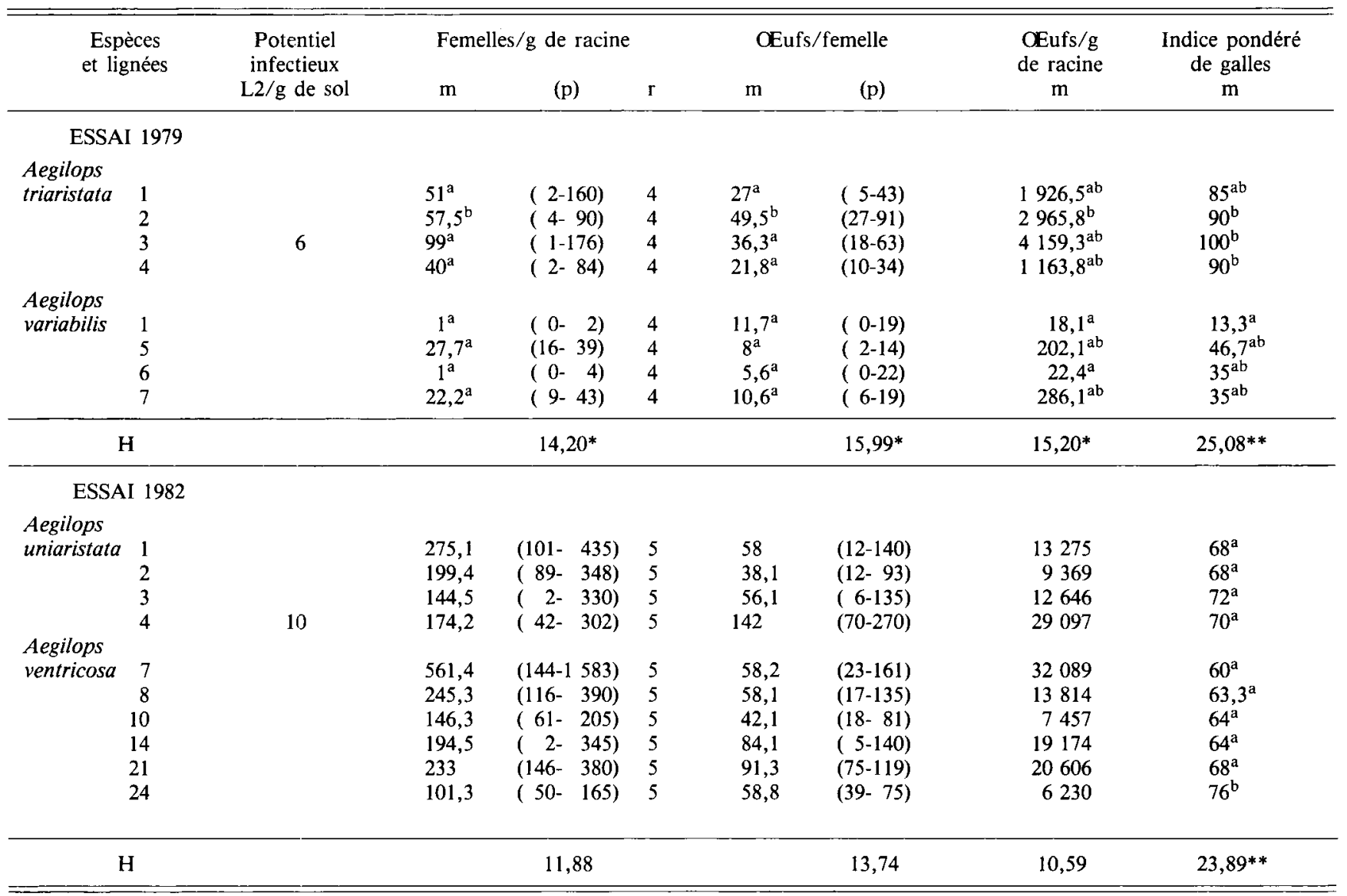

r, m, p, H : cf. tableau 1.

\section{DISCUSSION ET CONCLUSIONS}

Nos travaux démontrent le caractère multiplicateur vis-à-vis de $M$. naasi du blé diploïde $T$. $m$. beoticum et d'Ae. kotschyi jamais testés auparavant. Les blés diploïdes $T$. m. monococcum, $T$. urartu et les espèces sauvages $A e$. ventricosa et $A e$. uniaristata permettent la formation d'œufs du parasite et donc sa multiplication dans nos essais ainsi que dans ceux de RoBERTS et al. (1982) en Californie. Ae. triaristata, classé comme peu multiplicateur du nématode par les Britanniques (COOK et al., 1976) présente, comme les blés diploïdes, un caractère « bon hôte ». Il est difficile de comparer la multiplication du parasite sur ces différentes espèces puisque les essais n'ont pas tous été réalisés la même année. $T$. m. monococcum s'avère être cependant le moins bon hôte du nématode puisqu'il fournit effectivement moins de femelles et d'œufs par $\mathrm{g}$ de racine que $T$. m. beoticum lignée 1039 (tabl. 1), Ae. uniaristata, Ae. ventricosa en 1982 (tabl. 2) et que T. urartu, lignées 7796 et 7797 en 1980 (tabl. 1).

Ae. variabilis, contrairement aux résultats enregistrés par CoOK et al. (1976), présente dans nos essais des galles induites par le nématode ; ces dernières sont cependant de petite taille avec des diamètres souvent inférieurs ou égaux au $\mathrm{mm}$. Chacune des 4 lignées testées forme moins de femelles et d'œufs par $\mathrm{g}$ de racine que celles de Ae. triaristata (tabl. 2). Deux d'entre elles, $\mathrm{n}^{\text {os }} 1$ et 6 , présentent des plantes totalement résistantes à $M$. naasi; elles semblent d'autant plus intéressantes que DOSBA \& RIVOAL (1981) ont démontré leur caractère « mauvais hôte " vis-à-vis d'H. avenae.

Ae. umbellulata $(2 \mathrm{n}=14$, génome U) présente le même comportement que Ae. variabilis $(2 \mathrm{n}=28$, génomes US ${ }^{v}$ ) vis-à-vis de $M$. naasi et un bon niveau de résistance à $H$. avenae (RIVOAL, comm. pers.). Il apparaît vraisemblable que chez Ae. variabilis, le(s) gène(s) de résistance sont localisés sur le génome $U$. Cette hypothèse est confortée par le très bon niveau de multiplication chez Ae. speltoides dont le génome $\mathrm{S}$ est très proche de $\mathrm{S}^{\mathrm{v}}$.

Cette étude et celle de DosBA \& RivoAL (1981) montrent qu'on dispose avec $A e$. variabilis d'un géniteur de résistance à $M$. naasi et à $H$. avenae. L'introduction dans le blé tendre de ses gènes de résistance est envisagée. Des méthodes de cytogénétique ont été élaborées pour le transfert dans le blé tendre d'infor- 


\section{TABLEAU 3}

Etude de la résistance d'Aegilops variabilis Ae. kotschyi, Ae. umbellulata, Ae. speltoides à l'encontre de Meloidogyne naasi. Study of the resistance of Aegilops variabilis Ae. kotschyi, Ae. umbellulata, Ae. speltoides to Meloidogyne naasi.

\begin{tabular}{|c|c|c|c|c|c|c|c|c|c|c|}
\hline \multirow{2}{*}{\multicolumn{2}{|c|}{$\begin{array}{l}\text { Espèces } \\
\text { et lignées }\end{array}$}} & \multirow{2}{*}{$\begin{array}{c}\text { Potentiel } \\
\text { infectieux } \\
\text { L2/g de sol }\end{array}$} & \multicolumn{4}{|c|}{ Femelles/g de racine } & \multicolumn{2}{|c|}{ OEufs/femelle } & \multirow{2}{*}{$\begin{array}{c}\text { OEufs/g } \\
\text { de racine } \\
m\end{array}$} & \multirow{2}{*}{$\begin{array}{l}\text { Indice pondéré } \\
\text { de galles } \\
\text { m }\end{array}$} \\
\hline & & & $\mathrm{m}$ & & (p) & $\mathrm{r}$ & $\mathrm{m}$ & (p) & & \\
\hline \multicolumn{11}{|c|}{ ESSAI 1981} \\
\hline $\begin{array}{l}\text { Aegilops } \\
\text { variabilis }\end{array}$ & $\begin{array}{l}1 \\
5 \\
6 \\
7\end{array}$ & 22 & $\begin{array}{r}1,3^{\mathrm{a}} \\
89,9^{\mathrm{b}} \\
0,4^{\mathrm{a}} \\
31,8^{\mathrm{ab}}\end{array}$ & $\begin{array}{l}(0 \\
(18 \\
(0 \\
0\end{array}$ & $\begin{array}{l}-5) \\
-221) \\
-\quad 2) \\
-167)\end{array}$ & $\begin{array}{l}8 \\
8 \\
8 \\
8\end{array}$ & $\begin{array}{c}4,1^{\mathrm{ab}} \\
69,1^{\mathrm{c}} \\
1,2^{\mathrm{a}} \\
52,9^{\mathrm{abc}}\end{array}$ & $\begin{array}{l}(0-7) \\
(8-190) \\
(0-9) \\
(0-83)\end{array}$ & $\begin{array}{c}9,7^{\mathrm{a}} \\
3260^{\mathrm{b}} \\
1,8^{\mathrm{a}} \\
1323^{\mathrm{ab}}\end{array}$ & $\begin{array}{l}40^{\mathrm{a}} \\
77,5^{\mathrm{bc}} \\
55^{\mathrm{ab}} \\
75^{\mathrm{bc}}\end{array}$ \\
\hline $\begin{array}{l}\text { Aegilops } \\
\text { kotschyi }\end{array}$ & $\begin{array}{l}1 \\
2 \\
3 \\
4 \\
6 \\
\end{array}$ & & $\begin{array}{r}7,79^{\mathrm{ab}} \\
31,1^{\mathrm{ab}} \\
124,9^{\mathrm{b}} \\
8,8^{\mathrm{ab}} \\
48,5^{\mathrm{ab}}\end{array}$ & $\begin{array}{l}(0, \\
(3 \\
(8 \\
(1 \\
(3\end{array}$ & $\begin{array}{r}, 3-14) \\
-153) \\
-446) \\
-15) \\
-319)\end{array}$ & $\begin{array}{l}8 \\
8 \\
8 \\
8 \\
8\end{array}$ & $\begin{array}{c}26,1^{a b c} \\
18,6^{a b c} \\
45,9^{a b} \\
96,8^{c} \\
109,7^{c}\end{array}$ & $\begin{array}{l}(0-78) \\
(5-44) \\
(7-83) \\
(4-436) \\
(7-206)\end{array}$ & $\begin{array}{r}282^{\mathrm{ab}} \\
413^{\mathrm{ab}} \\
4536^{\mathrm{b}} \\
586^{\mathrm{ab}} \\
1172^{\mathrm{b}} \\
\end{array}$ & $\begin{array}{l}76,7^{\mathrm{bc}} \\
60^{\mathrm{abc}} \\
62,5^{\mathrm{abc}} \\
80^{\mathrm{bc}} \\
92,5^{\mathrm{c}}\end{array}$ \\
\hline \multicolumn{3}{|c|}{$\mathrm{H}$} & \multicolumn{5}{|c|}{$43,82^{* * *}$} & $35,1^{* * *}$ & $45,90^{* * *}$ & $33,41^{* * *}$ \\
\hline \multicolumn{11}{|c|}{ ESSAIS 1982 et 1984} \\
\hline $\begin{array}{l}\text { Aegilops } \\
\text { umbellulata }\end{array}$ & $\begin{array}{l}4 \\
5\end{array}$ & $\begin{array}{l}11 \\
10\end{array}$ & $\begin{array}{c}0,1^{\mathrm{a}} \\
0,3^{\mathrm{e}} \\
141,2^{\mathrm{ab}} \\
6,2^{\mathrm{ab}} \\
0,1^{\mathrm{e}} \\
366,4^{\mathrm{b}} \\
18,6^{\mathrm{ab}} \\
-3,8^{\mathrm{f}}\end{array}$ & 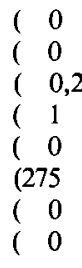 & $\begin{array}{rr}- & 0 \\
- & 1 \\
2- & 410 \\
- & 18 \\
- & 1 \\
- & 524 \\
- & 53 \\
- & 8\end{array}$ & $\left\{\begin{array}{l}5 \\
8 \\
5 \\
5 \\
7 \\
5 \\
5 \\
8\end{array}\right.$ & $\begin{array}{l}1^{\mathrm{a}} \\
4,8 \\
36 \\
13,6^{\mathrm{abc}} \\
40,5 \\
83,4^{\mathrm{c}} \\
6,3^{\mathrm{ab}} \\
30,3\end{array}$ & 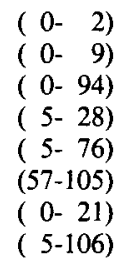 & $\begin{array}{c}7,2^{\mathrm{a}} \\
1^{\mathrm{e}} \\
10167^{\mathrm{ab}} \\
41,6^{\mathrm{a}} \\
8,2^{\mathrm{e}} \\
28581^{\mathrm{b}} \\
139^{\mathrm{a}} \\
160,9^{\mathrm{f}}\end{array}$ & $\begin{array}{l}60 \\
30 \\
64 \\
50 \\
42,9 \\
60 \\
50 \\
47,5\end{array}$ \\
\hline $\begin{array}{l}\text { Aegilops } \\
\text { speltoides }\end{array}$ & $\begin{array}{r}1 \\
3 \\
18 \\
25 \\
34\end{array}$ & & $\begin{array}{l}138,3^{\mathrm{ab}} \\
204,9^{\mathrm{ab}} \\
229,7^{\mathrm{b}} \\
361,6^{\mathrm{b}} \\
238,36^{\mathrm{b}}\end{array}$ & $\begin{array}{l}(14 \\
(173 \\
(40 \\
(44 \\
(59\end{array}$ & $\begin{array}{l}-\quad 319 \\
-\quad 256 \\
-\quad 434 \\
-1221 \\
-\quad 540\end{array}$ & $\begin{array}{l}5 \\
5 \\
5 \\
5 \\
5\end{array}$ & $\begin{array}{r}102,2^{\mathrm{bc}} \\
34,8^{\mathrm{abc}} \\
47,6^{\mathrm{abc}} \\
52,7^{\mathrm{abc}} \\
48,5^{\mathrm{abc}}\end{array}$ & $\begin{array}{l}(41-167) \\
(8-58) \\
(18-86) \\
(7-99) \\
(22-90)\end{array}$ & $\begin{array}{r}15193^{\mathrm{ab}} \\
9859^{\mathrm{ab}} \\
9390^{\mathrm{ab}} \\
23036^{\mathrm{ab}} \\
11197^{\mathrm{ab}}\end{array}$ & $\begin{array}{l}66,7 \\
60 \\
80 \\
48 \\
60\end{array}$ \\
\hline \multicolumn{3}{|c|}{$\mathrm{H}$} & & $\begin{array}{r}25, \\
-11,\end{array}$ & $\begin{array}{l}, 30^{* *} \\
07 * * *\end{array}$ & & & $\begin{array}{c}24,85^{* *} \\
5,17\end{array}$ & $\begin{array}{l}26,26^{* *} \\
10,98^{* * *}\end{array}$ & $\begin{array}{r}11,88 \\
4,92\end{array}$ \\
\hline
\end{tabular}

r, m, p, H : cf. tableau 1.

mations génétiques des Aegilops (LAW, 1981). De nombreux transferts de résistance à des maladies ont été réalisées (Sharma \& GiLl, 1983). Plus récemment, LAw (1983) a introduit dans le blé des gènes d'Ae. umbellulata codant pour des protéines du grain. Ceci confirme que si les gènes de résistance d'Ae. variabilis sont effectivement localisés sur les chromosomes du génome $\mathrm{U}$, leur introduction dans le blé peut être envisagée.

\section{RÉFÉRENCES BIBLIOGRAPHIQUES}

Bayon F., 1978. Les nématodes. Cultivar, 108, 92-93.

Caubel G., Ritter M., Rivoal R., 1972. Observations relatives à des attaques du nématode Meloidogyne naasi Franklin sur céréales et graminées fourragères, dans l'ouest de la France en 1970. $C . R$. Acad. Agric. Fr., 351-356.

Cook R., York P. A., 1981. Genetics of resistance to Heterodera avenae and Meloidogyne naasi. Proc. 4th Int. Barley Genet. Symp. Edinburgh. Edinburgh University Press, 418-424.

Cook R., York P. A., Chew B. H., 1976. Variation in host reaction to the cereal root knot nematode Meloidogyne naasi and new host records. Rep. Welsh Plant Breed. Stn. 71.
Cook R., York P. A., Evans J. L., 1981. Resistance in barley to Meloidogyne naasi. Rep. Welsh Plant Breed. Stn for 1981, 92.

Coolen W. A., d'Herde C. J., 1972. A method for the quantitative extraction of nematodes from plant tissue. Publ. Gouv. Res. St. Nematol. Entomol., Merelbecke, Belgium, 1-77.

Dosba F., Rivoal R., 1981. Les lignées d'addition blé-Aegilops ventricosa Tausch II. Etude de leur comportement et de celui de leurs progéniteurs vis-à-vis d'Heterodera avenae Woll. Agronomie, 1, 559-564.

Dunn O. J., 1964. Multiple comparisons using rank sums. Technometrics, 6, 241-252. 
Furuta Y., 1981. Intraspecific variation in Aegilops variabilis and Aegilops kotschyi revealed by chromosome pairing in F1 hybrids. Jap. J. Genet., 56, 495-504.

Hollander M., Wolfe D. A., 1973. Non Parametric Statistical Methods. J. Wiley and sons Inc., $503 \mathrm{p}$.

Law C. N., 1981. Chromosome manipulation in wheat. Chromosomes today, 7, 194-205.

Law C. N., 1983. Transfer of genes for grain protein type from Aegilops umbellulata into wheat. Rep. Plant. Breed. Inst., 58-59.

Person-Dedryver F., 1984. Les céréales à paille, hôtes de Meloidogyne naasi Franklin. I. - Mise au point de méthodes et résultats préliminaires d'évaluation de la résistance ou du caractère multiplicateur. Agronomie, 4 (10), 977-985.
Person-Dedryver F., 1985. Les céréales à paille, hôtes de Meloidogyne naasi Franklin. II. - Variabilité du comportement multiplicateur ou résistant de variétés cultivées en France. Agronomie, 5 (1), 55-62.

Rivoal R., Dedryver F., Caubel G., 1983. Céréales : possibilités de lutte contre les nématodes. Phytoma, 348, 11-13.

Roberts P. A., Van Gundy S. D., Waines J. G., 1982. Reaction of wild and domesticated Triticum and Aegilops species to root knot nematodes (Meloidogyne). Nematologica, 28, 182-191.

Sharma H. C., Gill B. S., 1983. Current status of wide hybridization in wheat. Euphytica, 32, 17-32. 